\title{
Pre-coded Orthogonal Space-Time Block Codes over MIMO Channel and its Analysis for Antenna Selection Technique
}

\author{
Priya Dhawan \\ Student, Deptt.of ECE \\ Amritsar College of Engg \& Technology \\ Amritsar-143001, Punjab, India
}

\author{
Narinder Sharma \\ HOD, Deptt.of EEE \\ Amritsar College of Engg \& Technology \\ Amritsar-143001, Punjab, India
}

\begin{abstract}
A pre-coder is designed for orthogonal space-time block codes (OSTBCs) in multiple-input multiple-output (MIMO) channels. Unlike previous works, our pre-coder is the first to be designed to minimize the exact symbol error rate. In this paper, we show that OSTBCs can achieve full diversity and nearly strict orthogonality with a small amount of feedback bits returned from the receiver back to the transmitter. Along with pre-coding of the OSTBC, also the proper utilization of partial CSI is done at the transmitter side to improve overall characteristics of the system. Simulation results of the precoding OSTBC is discussed along with its antenna design technique.
\end{abstract}

\section{General Terms}

Channel Equalization, MIMO-OFDM system, Channel State Information

\section{Keywords}

MIMO systems, RF modules, Antenna Selection, Channel State Information, Signal to Noise Ratio

\section{INTRODUCTION}

In typical digital communication system, MIMO stands for multiple-input multiple-output means multiple antennas at both link ends of a communication system, i.e., at the transmit and at the receive side. The multiple-antennas at the transmitter and/or at the receiver in a wireless communication link open a new dimension in reliable communication, which can improve the system performance substantially. The idea behind MIMO is that the transmit antennas at one end and the receiver antennas at the other end are "connected and combined" in such a way that the quality (the bit error rate (BER), or the data rate) for each user is improved. The core idea in MIMO transmission is space-time signal processing in which signal processing in time is complemented by signal processing in the spatial dimension by using multiple, spatially distributed antennas at both link ends.

The OSTBC Encoder block encodes an input symbol sequence using orthogonal space-time block code (OSTBC). The block maps the input symbols block-wise and concatenates the output codeword matrices in the time domain. [2]

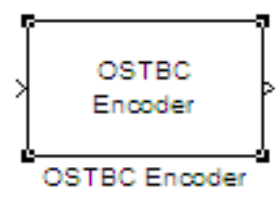

Fig. 1.OSTBC Encoder [2]

It is the block type representation of OSTBC and it supports time and spatial domains for OSTBC transmission. It also supports an optional dimension, over which the encoding calculation is independent. This dimension can be thought of as the frequency domain. The following illustration indicates the supported dimensions for the inputs and output of the OSTBC Encoder block [2].

\section{USING PRE-CODED OSTBC IN SYSTEMS}

Let us have a MISO system with $\mathrm{N}_{\mathrm{T}}$ antennas, that is, $h \in \mathfrak{R}^{1 \times N_{T}}$. Let $C \in \mathfrak{R}^{M \times T}$ denote a space time codeword with a length of $\mathrm{M}$, and is represented as

$$
C=\left[c_{1} c_{2} \cdots c_{T}\right]
$$

Where $c_{k}=\left[c_{k, 1} c_{k, 2} \cdots c_{k, M}\right]^{T}, k=1,2, \cdots, T$, and $M \leq N_{T}$. In the pre-coded OSTBC systems, the spacetime codeword $\mathrm{C}$ is multiplied by a pre-coding matrix $W \in \mathfrak{R}^{N_{T} \times M}$, where $\mathrm{W}$ is chosen from the code book $F=\left\{W_{1}, W_{2}, W_{3}, \cdots, W_{L}\right\} . \mathrm{W}$ is chosen such that the codeword improves channel capacity or error performance. Assuming that $\mathrm{N}_{\mathrm{T}}$ channels remain static over $\mathrm{T}$, the received signal $y \in \mathfrak{R}^{l \times T}$ can be expressed as

$$
y=\sqrt{\frac{E_{X}}{N_{T}}} h W C+z
$$


In above Equation, the length of each vector is $M \leq N_{T}$ while the space time codeword is composed of $\mathrm{T}$ column vectors. For a given channel $\mathrm{h}$ and pre-coding matrix $\mathrm{W}$, we consider the pair wise codeword error probability $\operatorname{Pr}\left(C_{i} \rightarrow C_{j} \mid H\right)$ [3].This is the probability that the space-time codeword $\mathrm{C}_{\mathrm{i}}$ is transmitted whereas $\mathrm{C}_{\mathrm{j}}$ with $j \neq i$ is decoded. After few mathematical manipulations, the upper bound of the pair wise error probability can be derived as

$$
\operatorname{Pr}\left(C_{i} \rightarrow C_{j} \mid H\right)=Q\left(\sqrt{\frac{\rho\left\|H W E_{i, j}\right\|_{F}^{2}}{2 N_{T}}}\right) \leq \exp \left(-\frac{\rho\left\|H W E_{i, j}\right\|_{F}^{2}}{4 N_{T}}\right)
$$

Where $\rho$ is the signal-to-noise ratio (SNR) and is given as $\rho=E_{X} / N_{0}$, and $E_{i . j}=C_{i}-C_{j}$ is the error matrix between the codewords $C_{i}$ and $C_{j}$. From above Equation, we can interpret that in order to minimize the pair wise error probability [9, 10] $\left\|H W E_{i . j}\right\|_{F}^{2}$ needs to be maximized, which leads us to the following codeword selection criterion

$$
\begin{aligned}
W_{o p t}=\underset{W \in F, i \neq j}{\arg \max }\left\|H W E_{i . j}\right\|_{F}^{2} \\
=\underset{W \in F, i \neq j}{\arg \max } \operatorname{Tr}\left(H W E_{i . j} E_{i . j}^{H} W^{H} H^{H}\right) \\
=\underset{W \in F}{\arg \max } \operatorname{Tr}\left(H W E^{H} H^{H}\right) \\
=\underset{W \in F}{\arg \max }\|H W\|_{F}^{2}
\end{aligned}
$$

The optimum solution of can be given by

$$
W_{o p t}=\left[V_{1} V_{2} \cdots V_{M}\right] . \Delta \bar{V}
$$

Since $\bar{V}$ is unitary, $\lambda_{i}\left(W_{\text {opt }}\right)=1, \mathrm{i}=1,2, \cdots, \mathrm{M}$,

where $\lambda_{i}(A)$ denotes the $\mathrm{i}^{\text {th }}$ largest eigen value of the matrix ujh AA. For not deterministic channel codebook can be designed as

$$
E\left\{\min _{W \in F}\left(\left\|H W_{o p t}\right\|_{F}^{2}-\|H W\|_{F}^{2}\right)\right\}
$$

The above expected value in Equation is upper-bounded as

$$
E\left\{\min _{W \in F}\left(\left\|H W_{\text {opt }}\right\|_{F}^{2}-\|H W\|_{F}^{2}\right)\right\} \leq E\left\{\lambda_{1}^{2}\{H\}\right\} E\left\{\left\|\bar{V} \bar{V}^{H}-W W^{H}\right\|_{F}^{2}\right\}
$$

Since $\lambda_{1}^{2}\{H\}$ is given, the codebook must be designed so as to minimize $\mathrm{E} \min \mathrm{W}_{\mathrm{opt}}$. The corresponding minimization problem can be formulated into the Grassmannian subspace packing problem [5]. The in Grassmannian subspace packing is the chordal distance is used as performance measure and is defined as

$$
d\left(W_{k}, W_{l}\right)=\frac{1}{\sqrt{2}}\left\|W_{k} W_{k}^{H}-W_{l} W_{l}^{H}\right\|_{F}
$$

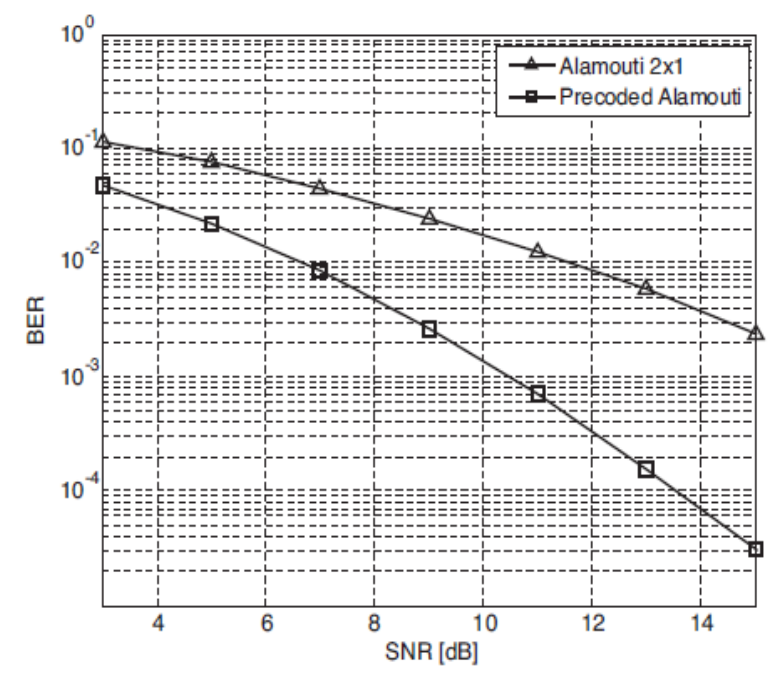

Fig 2. BER performance of OSTBC with and without precoding in Rayleigh fading channel

For random channels, the optimum codebook is designed so as to maximize the minimum chordal distance $\delta_{\min }=\min _{k \neq l, 1 \leq k, l \leq L} d\left(W_{k}, W_{l}\right)$

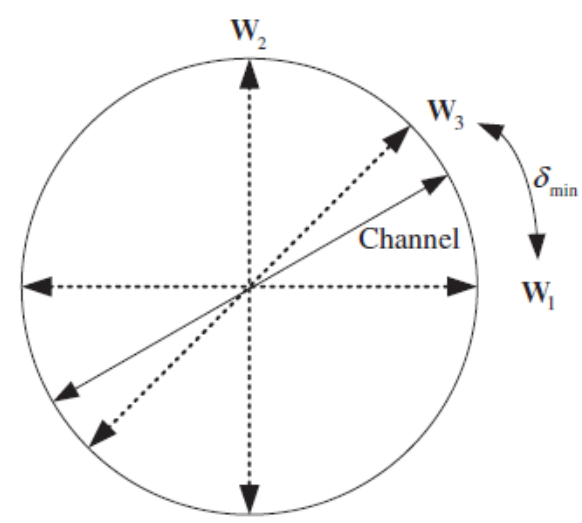

Figure 3. Precoding matrix and chordal distance 
For $\mathrm{N}_{\mathrm{T}}$, codeword length $\mathrm{M}$, and codebook size $\mathrm{L}$ Grassmannian packing problem can be solved as

$$
F=\left\{W_{D F T}, \theta W_{D F T}, \cdots, \theta^{L-1} W_{D F T}\right\}
$$

Where $\mathrm{u}$ is the diagonal matrix and is given as

$$
\theta=\operatorname{diag}\left(\left[e^{j 2 \pi u_{1} / N_{T}} e^{j 2 \pi u_{2} / N_{T}} \cdots e^{j 2 \pi u_{N_{T}} / N_{T}}\right]\right)
$$

IEEE 802.16e specification for the Mobile WiMAX system uses this $\mathrm{r}$ design method. When $N_{T}=4, M=3$, and $L=64, W_{1}$ is given as

$$
W_{1}=\frac{1}{\sqrt{4}}\left[\begin{array}{ccc}
1 & 1 & 1 \\
1 & e^{j 2 \pi \cdot 1 \cdot 2 / 4} & e^{j 2 \pi \cdot 1 \cdot 3 / 4} \\
1 & e^{j 2 \pi \cdot 2 \cdot 2 / 4} & e^{j 2 \pi \cdot 2 \cdot 3 / 4} \\
1 & e^{j 2 \pi \cdot 3 \cdot 2 / 4} & e^{j 2 \pi \cdot 3 \cdot 3 / 4}
\end{array}\right]
$$

The remaining precoding matrices $\mathrm{W}_{\mathrm{i}}$ are obtained as

$$
W_{i}=\operatorname{diag}\left(\left[e^{j 2 \pi \cdot 1 / 4} e^{j 2 \pi \cdot 8 / 4} e^{j 2 \pi \cdot 61 / 4} e^{j 2 \pi \cdot 45 / 4}\right]\right)^{i-1} w_{1}, i=2,3, \cdots, 64
$$

\section{ANTENNA SELECTION TECHNIQUE FOR OSTBC}

Gain is the major parameter that is considered to calculate overall system performance. The simulation is explained in this section.The antenna selection technique is one of the major issues that is to be taken care in the communication system. MIMO systems have better performance which can be achieved without using additional transmit power or bandwidth extension. But during simulation it is seen that diversity gain has been achieved without using additional RF modules on the transmitter side.To simulate the antenna selection method for the Alamouti STBC scheme has been used to yield its BER performance with $\mathrm{Q}=2$ and $\mathrm{N}_{\mathrm{T}}=4$. Note that the further diversity gain has been achieved without using additional RF modules on the transmitter side. After comparing the results, it is demonstrated that the antenna selection method provides more gain over the pre-coding method.

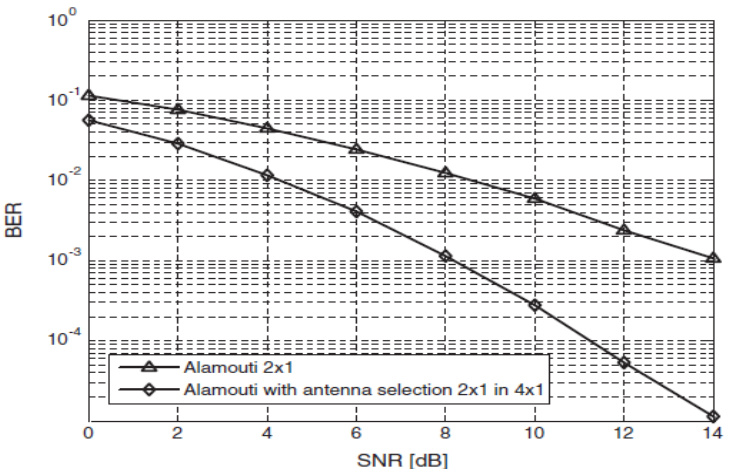

Fig.4. BER performance of Alamouti STBC scheme with antenna selection: $Q=2$ and $N_{T}=4$
To reduce the cost associated with the multiple RF modules, antenna selection techniques can be used to employ a smaller number of RF modules than the number of transmit antennas. Figure 5 illustrates the end-to-end configuration of the antenna selection in which only Q RF modules are used to support $\mathrm{N}_{\mathrm{T}}$ transmit antennas $\left(Q<N_{T}\right)$ and $\mathrm{Q} \mathrm{RF}$ modules are selectively mapped to $\mathrm{Q}$ of $\mathrm{N}_{\mathrm{T}}$ transmits antennas.

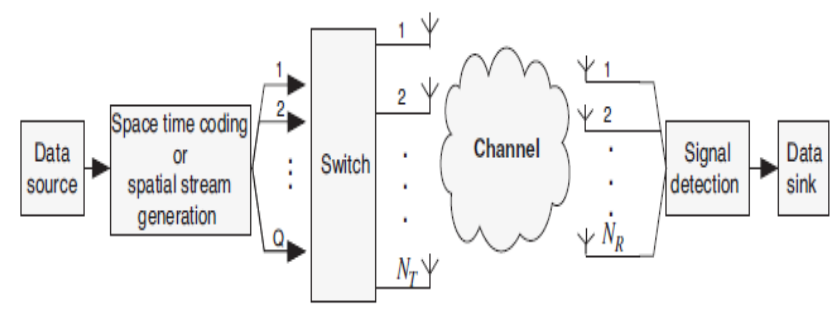

Figure 5: Antenna selections with $Q \mathbf{R F}$ modules and $\mathbf{N}_{\mathrm{T}}$ transmit antennas $\left(Q<N_{T}\right)[8]$

\section{CONCLUSION}

In this paper, The Orthogonal Space-Time Block Codes are discussed over MIMO and MISO channels. It is seen that the OSTBC pre-coder is when used the overall parameters of the system gets improved. Besides the other two techniques of antenna selection that are optimal antenna technique and complexity reduced antenna selection technique, the OSTBC antenna selection technique is discussed, we adopted an approach in which additional antenna is selected in ascending order of increasing the channel capacity i.e. one antenna with the highest capacity is first selected we have used transmission techniques that can be used to exploit the CSI on the transmitter side. Simulation results show that the channel capacity increases in proportion to the number of the selected antennas. It is concluded that the use of Pre-coder OSTBC and the encoding of an input symbol sequence using orthogonal space-time block code increases system performance and at receiver the results achieved are much better.

\section{REFERENCES}

[1] Reduced-Rank Estimation Of Non stationary TimeVariant Channels Using Subspace Selection. L. H. Xing, Zh. H. Yu, Zh. P. Gao, And L. Zha (2006)Channel Estimation For Transmitter Diversity OFDM Systems. $1^{\text {st }}$ IEEE Conference on Digital Object Identifier (2006). Page: $1-4$.

[2] OSTBC Encoder - MathWorks: http://www.mathworks.in/help/comm/ref/ostbcencoder.ht $\mathrm{ml}$

[3] Jun Shikida, Satoshi Suyama, Hiroshi Suzuki, and Kazuhiko Fukawa (2010). Iterative Receiver Employing Multiuser Detection and Channel Estimation for MIMOOFDMA. IEEE 71st Vehicular Technology Conference (2010). Page: 1-5.

[4] Bharath B. N. and Chandra R. Murthy. (2012) Channel Estimation At The Transmitter In a Reciprocal MIMO Spatial Multiplexing System. IEEE National Conference (2012), Page: 1-5. 
[5] JiaMeng, Wotao Yin, Yingying Li, Nam Tuan Nguyen, and Zhu Han (2012). IEEE Journal Of Selected Topics In Signal Processing, 6(1) February 2012. Page: 15-25.

[6] Thomas Zemen, and Andreas F. Molisch, (2012) Adaptive IEEE Transactions, 61(9) (2012). Page: 40424056

[7] Baoguo Yang, Khaled Ben Letaief, Roger S. Cheng and Zhigang Cao(March 2001), Channel Estimation For OFDM Transmission In Multipath Fading Channels Based On Parametric Channel Modeling, IEEE Transactions On Communications, Vol. 49.

[8] Juinn-Horng Deng And Yi-Hsin Lint (2013). An Iterative Receiver Design WithCfo, Sfo, Channel Estimation And
Equalization For OFDM Systems Over Multipath Fading Channels. 15th International Conference on Advanced Communication Technology (Icact), 2013. Page: 233 237.

[9] Larsson, E.G., Ganesan, G., Stoica, P., and Wong, W.H. (2002) On the performance of orthogonal spacetimeblock coding with quantized feedback. IEEE Commun. Letters, 12(6), 487-489.

[10] Love, D.J. and Heath, R.W. Jr (2005) Limited feedback unitary precoding for orthogonal space-time block codes IEEE Trans. Signal. Proc., 53(1), 64-73. 